\title{
DOR OROFACIAL, DISFUNÇÃO TEMPOROMANDIBULAR E OSTEOPOROSE SENIL: ABORDAGEM ODONTOLÓGICA EM IDOSOS
}

\author{
OROFACIAL PAIN, TEMPOROMANDIBULAR DISORDER AND SENILE \\ OSTEOPOROSIS: DENTAL APPROACH IN ELDERLY
}

Sérgio Spezzia

Cirurgião Dentista e Mestre em Ciências pela Escola Paulista de Medicina Universidade Federal de São Paulo.

\section{Autor Principal:}

Sérgio Spezzia

Email: sergio.spezzia@unifesp.br 


\section{Resumo}

Dor orofacial (DOF), consta do conjunto de condições dolorosas provenientes da boca e face, incluindo dor de dente, disfunções temporomandibulares (DTMs), neuralgias, alguns tipos de cefaleias e outros quadros dolorosos. Osteoporose é uma doença osteometabólica caracterizada por perda de massa óssea e deterioração da microarquitetura do tecido ósseo com consequente fragilidade óssea e maior suscetibilidade à fraturas. Pode-se classificar a osteoporose em osteoporose senil e osteoporose pós-menopausa. Algumas alterações sistêmicas, como a osteoporose podem provocar repercussões nas DOF. O objetivo proposto no presente artigo foi evidenciar como a osteoporose senil pode agir a nível da saúde bucal dos indivíduos, afligindo possivelmente a articulação temporomandibular (ATM) dos mesmos, ocasionando DTM, com manifestações nas DOF. Alterações sistêmicas que comprometem outras articulações do corpo podem atingir a ATM, é o que ocorre nos casos da artrite psoriatica, lúpus e na artrite reumatoide. Algumas dessas alterações, tal como a osteoporose podem ter correlação com DOF e DTM e devem ter diagnóstico diferencial e tratamento odontológico diferenciado. $\mathrm{O}$ acometimento por osteoporose na ATM pode ter diagnóstico de DTM. Concluiu-se que a osteoporose pode influir nas manifestações clínicas das DOF, sendo fundamental o conhecimento e a conscientização pelo cirurgião dentista dessa doença sistêmica que pode acometer a saúde bucal dos seus pacientes.

Palavras-chave: Idoso. Osteoporose. Dor Facial. Palpação.

Keywords: Aged. Osteoporosis. Orofacial Pain. Palpation. 


\section{INTRODUÇÃO}

A osteoporose é uma doença osteometabólica caracterizada por perda de massa óssea e deterioração da microarquitetura do tecido ósseo com consequente fragilidade óssea e maior suscetibilidade à fraturas. Pode-se classificar a osteoporose em osteoporose senil e osteoporose pós-menopausa (WHO, 1994; NATIONAL OSTEOPOROSIS FOUNDATION, 2010).

Em idosos convive-se frequentemente com a presença de osteoporose, que aflige em maior escala o gênero feminino. Mulheres no período pós-menopausa são acometidas, bem como indivíduos idosos do gênero masculino (WHO, 1994).

A osteoporose pós-menopausa aflige comumente mulheres com faixa etária entre 50 e 70 anos, nessa situação têm-se redução de massa óssea, havendo comprometimento maior no osso trabecular. Já a osteoporose senil acomete indivíduos com faixa etária posterior aos 65 e 70 anos, havendo redução de massa óssea, advinda da redução de resposta hormonal e nutricional inerente a osteoblastos e osteoclastos, em decorrência da presença da senilidade. Na osteoporose senil, têm-se desmineralização óssea tanto no osso cortical quanto no trabecular (WHO, 1994; NATIONAL OSTEOPOROSIS FOUNDATION, 2010).

Entende-se por dor orofacial (DOF), o conjunto de condições dolorosas provenientes da boca $\mathrm{e}$ face, incluindo a dor de dente, as disfunções temporomandibulares (DTMs), as neuralgias, alguns tipos de cefaleias e outros quadros dolorosos (SIQUEIRA, JTT, 2001). Dentre as DOF uma das causas mais prevalentes são as DTMs, constituindo um grupo de condições dolorosas que provocam dores crônicas na região da cabeça, articulação temporomandibular (ATM), músculos mastigatórios, região sub-occipital e musculatura supra-escapular e que atingem cerca de 6\% da população brasileira (SIQUEIRA, JTT, 2005). Existe uma classificação das mesmas, em musculares, articulares e mistas (SIQUEIRA, JTT \& TEIXEIRA, MJ, 1998). 
A ATM pode ser afetada por uma ampla variedade de doenças e a maioria delas pode criar sintomas de dor e disfunção. Esses sintomas podem assemelhar-se aos atribuídos para problemas dos músculos mastigatórios primários, chamados de dordisfunção miofacial (MOHL, ND et al., 1991).

Alterações sistêmicas que comprometem outras articulações do corpo podem atingir a ATM, é o que ocorre nos casos da artrite psoriatica, lúpus e na artrite reumatoide (MOLINA, OF, 1989). Algumas dessas alterações, tal como a osteoporose podem ter correlação com DOF e DTM e devem ter diagnóstico diferencial e tratamento odontológico diferenciado. Nestes casos, a queixa principal relatada pelos pacientes é um quesito de extrema importância (TURP, JC et al., 1997; KLINEBERG, I et al., 1998). Sabe-se que o acometimento de osteoporose na ATM atualmente pode ter diagnóstico de DTM (SPEZZIA, S, 2013).

O objetivo proposto no presente artigo foi evidenciar como a osteoporose senil pode agir a nível da saúde bucal dos indivíduos, afligindo possivelmente a ATM dos mesmos, ocasionando DTM, com manifestações de DOF.

\section{REVISÃO DE LITERATURA}

A dor é considerada uma experiência desagradável, sensitiva e emocional, associada ou não ao dano real ou potencial de lesões dos tecidos, relacionando-se com a memória individual, expectativas e emoções de cada pessoa, podendo ser aguda ou crônica. Frequentemente a dor está associada a disfunção do sistema mastigatório (PEDROSO, RA \& CELICH, KLS, 2006; COHEN, LL et al., 2008).

A ATM normal move-se livremente sem desprender esforços intensos e sem desencadear sintomatologia dolorosa (TOMMASI, AF, 2002).

Sabe-se que, tanto músculos como articulações saudáveis não apresentam sinal de dor no desempenho de sua função normal ou quando palpados, entretanto se a dor estiver presente ao toque, significa sinal de que o músculo ou a ATM não estão equilibrados (LOBO, LF \& NUNES, LJ, 2000). 
Nos distúrbios da ATM ocorre dor, cuja etiologia deve envolver para sua determinação, análise das estruturas ósseas, muscular, ligamentosa e capsular da região da articulação, bem como o comportamento fisiológico apresentado por estas partes.

Ao examinar pacientes com disfunção da ATM, deve-se considerar os sintomas presentes dentro de um panorama geral das manifestações patológicas, chegando posteriormente por exclusão a um diagnóstico diferencial.

O diagnóstico de distúrbios da ATM deve ser realizado a partir de história completa, de exame físico detalhado, bem como com o uso de exames complementares. Na ATM, facilmente uma disfunção pode ser identificada, no entanto, a obtenção subsequente do diagnóstico diferencial e da terapêutica adequada a ser aplicada são bastante complexos. Exige-se para que se obtenha êxito, uma compreensão dos mecanismos fisiológicos que atuam na dor facial e nos distúrbios temporomandibulares (SIQUEIRA, JTT, 2001; TOMMASI, AF, 2002).

Por volta dos quarenta anos de idade, inicia-se um processo lento e progressivo de perda óssea, resultando eventualmente no desenvolvimento de osteoporose senil, caracterizando-se pela redução da densidade mineral óssea e perda do conteúdo mineral (NATIONAL OSTEOPOROSIS FOUNDATION, 2010).

Pacientes portadores de osteoporose senil devem receber atenção especial, no que tange aos cuidados odontológicos realizados no transcorrer das condutas clínicas, uma vez que as manifestações de osteoporose podem ocorrer também em âmbito bucal, agindo concomitantemente a outras manifestações clínicas presentes. Idosos por si só geralmente requerem maiores cuidados odontológicos, devido aos problemas bucais, que comumente os acometem, como cárie dentária, doenças periodontais e perdas dentárias. Além disso, pode haver manifestações da osteoporose senil em idosos que podem repercutir em âmbito bucal, a nível de ATM, podendo ocasionar manifestações de DTM e DOF. A realização de uma investigação detalhada pode averiguar se a osteoporose está agindo concomitantemente (SPEZZIA, S, 2013).

Várias doenças sistêmicas podem acometer o sistema estomatognático, acarretando o seu desequilíbrio ou mau funcionamento, dentre as quais, a osteoporose. Existe comprometimento da osteoporose a nível sistêmico e também em outras localidades, podendo a mesma afligir a região da face e suas adjacências. Nos ossos 
maxilares pode ocorrer também osteopenia e osteoporose (SPEZZIA, S, 2013; SPEZZIA, S, 2014; SPEZZIA, S, 2016; SPEZZIA, S, 2017).

Nesse contexto, e inerente a possível presença da osteoporose no sistema estomatognático, sabe-se que no geral, têm-se envelhecimento com o passar dos anos em todas estruturas do organismo, incluindo as localizadas na cavidade bucal, onde ocorrem muitas alterações, que levam possivelmente a instalação de alterações funcionais e facilitam o acometimento por doenças. Cemento e osso alveolar com o avançar da idade, apresentam alterações, como: diminuição da capacidade metabólica de cicatrização; menor vascularização e possível presença de osteoporose (SPEZZIA, S, 2017).

As manifestações ósseas bucais da osteoporose envolvem possivelmente, acometimento por disfunção temporomandibular; redução do rebordo alveolar; redução da massa e densidade óssea maxilar; edentulismo; diminuição da espessura óssea cortical e o acometimento por problemas periodontais, entre outras ocorrências (SPEZZIA, S, 2017).

A osteoporose pode afetar a ATM e contribuir para a reabsorção dos componentes condilar e temporal, o que poderia levar à sua disfunção e a fraturas patogênicas (DERVIS, E, 2005).

As DTMs são comumente encontradas em idosos, porém, nem sempre fáceis de avaliar. No doente idoso com DOF a abordagem clínica almeja identificar a sua queixa principal (SIQUEIRA, JTT \& JACOBSEN, MT, 2001).

Dos problemas bucais do idoso, a perda de dentes é um dos mais frequentes. Em decorrência disso, a reabilitação oral protética, torna-se importante para o restabelecimento das condições bucais.

Na semiotécnica da DOF, exames auxiliares e complementares são necessários durante avaliação do idoso, particularmente em desdentados totais. Na semiotécnica óssea, o exame do paciente acometido por doença óssea não difere em nada da semiotécnica empregada para doenças de outros tecidos.

Procede-se ao preenchimento de ficha clínica, contendo informações sobre a história médica e odontológica dos idosos. A história pregressa é quesito importante que 
deve incluir dados médicos ocupacionais, emocionais, sociais e familiares. Em quaisquer pacientes com DTM e dor, convém levantar sua história, cuidadosamente.

No decorrer do exame clínico do sistema mastigatório, deve-se proceder a observação de todo o corpo, podendo dessa forma, obter-se dados úteis no diagnóstico correto, principalmente ao analisarmos pacientes com doenças musculares e articulares.

O exame deve abranger ATMs, músculos mastigatórios, mobilidade mandibular, tecidos orais, dentição e oclusão e outras estruturas da cabeça e do rosto, relacionadas com a função do sistema mastigatório.

A queixa principal é o motivo que leva o paciente à procura do profissional de saúde. A queixa sempre que possível deve ser registrada na ficha clínica, utilizando-se das próprias palavras do paciente (SIQUEIRA, JTT, 2001; TOMMASI, AF, 2002).

A história médica visa obter informações detalhadas acerca das doenças sistêmicas que afligiram os pacientes no decorrer de suas vidas. O levantamento desses dados pode ser de relevância clínica em termos diagnósticos, prognósticos e terapêuticos, uma vez que contém o quadro geral de saúde e de doença em determinado indivíduo, considerando todo seu período de vida (SIQUEIRA, JTT, 2001; TOMMASI, $\mathrm{AF}, 2002)$.

O exame físico utiliza-se dos sentidos naturais para explorar os sinais, sendo realizado por intermédio de algumas manobras, entre elas a palpação. A palpação deve abranger o conjunto das estruturas extra e intrabucais, incluindo as estruturas mais afastadas que podem ou não possuir alterações teciduais. Através dela, pode-se obter informações sobre a consistência, limites, sensibilidade, textura superficial, infiltração, pulsação, flutuação, mobilidade e temperatura das lesões. Na boca, por intermédio da palpação, pode-se acessar quase todas as áreas importantes da boca, face e pescoço (SIQUEIRA, JTT, 2001; TOMMASI, AF, 2002).

O exame físico deve ser feito minuciosamente, concomitante a análise detalhada da história médica e odontológica dos pacientes, envolvendo a interpretação do estado da anatomia e fisiologia mandibulares, constituindo assim, forma de se obter importantes fontes de sintomas (SIQUEIRA, JTT, 2001; TOMMASI, AF, 2002).

A parte mais importante do exame da ATM é a palpação, que fornece informações sobre a sensibilidade e sobre os movimentos condilares. A palpação da 
musculatura faz-se sob duas formas: extrabucal e intrabucal. A musculatura é efetivamente examinada pela palpação. Através dela, pode-se avaliar a sensibilidade, o tamanho, a consistência e a rigidez dos músculos. Tipicamente, os lados direito e esquerdo são comparados durante a palpação de cada par de músculos (SIQUEIRA, JTT, 2001; TOMMASI, AF, 2002).

É possível a avaliação individual da função articular através da medição de vários movimentos mandibulares, como abertura, fechamento, movimento lateral, protusivo e retrusivo. A variação da movimentação da mandíbula é um guia importante para avaliação da capacidade funcional do sistema mastigatório. As medições dos movimentos horizontais e verticais máximos, como abertura, lateralidade e protusão devem ser realizados. São registrados os valores máximos, independentemente dos sintomas (movimentos realizados com dor ou não concomitantemente), bem como os locais onde incide dor ou desconforto pela primeira vez. Recomenda-se, ainda, que qualquer diferença entre um movimento ativo e um passivo, respectivamente movimento realizado voluntariamente pelo paciente e outro obtido após estiramento pelo examinador seja avaliado. Os desvios de linha média, ruídos e dor, bem como a capacidade de movimento passivo e ativo e as diferenças entre a excursão direita e esquerda devem ser todos observados. Estes serão levados em consideração, posteriormente na avaliação realizada conjuntamente com outros achados clínicos (MOHL, ND et al., 1991).

Os movimentos mandibulares são aferidos com a utilização de alguns possíveis meios auxiliares, como: régua milimetrada, fita métrica ou paquímetro (BARROS, LF, 2004).

A auscultação dos sons da articulação pode ser realizada com estetoscópio, porém a combinação da audição e da palpação, geralmente, faz-se suficiente para caracterizar ruídos, envolvendo estalos ou crepitação.

O estalo é o ruído que aparece, devido ao movimento não coordenado entre o menisco e o côndilo. O músculo responsável pelo funcionamento harmônico destas duas partes é o pterigóideo externo. O estalo é o ruído causado pelo choque do côndilo com a borda do menisco durante a abertura e fechamento da boca e é um dos primeiros sinais da DTM. 
A crepitação é um tipo de ruído mais frequente em pessoas idosas, ficando evidenciado com sua presença, o processamento de um fenômeno degenerativo da ATM, que envolve principalmente a superfície dos côndilos (MOHL, ND et al., 1991; TOMMASI, AF, 2002).

Os movimentos mandibulares podem ser desdobrados em séries de deslocamentos, que ocorrem ao redor dos eixos horizontal, vertical e sagital. Os movimentos mandibulares são a combinação de rotações ao redor de vários eixos. A abertura e o fechamento são a combinação de dois movimentos. Ocorre um movimento de rotação puro produzido pelos côndilos, girando no compartimento inferior da ATM e no compartimento superior ocorre movimento de translação ou deslizamento. Quando a mandíbula desliza para frente, promovendo contato borda a borda entre os dentes da maxila e mandíbula, produz-se posição protusiva. A movimentação da mandíbula lateralmente coloca esse lado em posição de trabalho e o lado contrário em posição de balanceio (MOHL, ND et al., 1991).

Quando os dentes não se encontram em harmonia com a articulação e os movimentos da mandíbula, ocorre interferências, que são contatos oclusais indesejáveis, possíveis causadores de desvios, indo desde o fechamento até a máxima intercuspidação ou atrapalhando a passagem para a posição de intercuspidação. Esses dados devem ser anotados na ficha clínica do paciente, juntamente com os demais achados.

Para exame clínico da função mandibular e do funcionamento do sistema mastigatório, faz-se algumas verificações. A abertura de boca ou mandibular, medida em milímetros, é realizada em abertura máxima (ativa) sem dor, configurando o grau máximo de abertura mandibular passível de ser executado pelo paciente sem que ocorra desconforto. Quando a medida desta abertura estiver entre 35 e $55 \mathrm{~mm}$, pode-se considerar uma abertura normal. O desvio da mandíbula em abertura ou fechamento é dado de valia na determinação diagnóstica. Movimentos de abertura e protusivo também são observados quanto a irregularidades e desvio e as diferenças entre os movimentos nos lados direito e esquerdo são evidenciadas (MOHL, ND et al., 1991).

\section{DISCUSSÃO}


Convém frisar que, a abordagem odontológica, tanto em indivíduos com osteoporose pós-menopáusica como nos com osteoporose senil, faz-se muito importante, devido as implicações em âmbito bucal ocasionadas por ambas doenças, entretanto, no presente artigo optou-se por evidenciar as repercussões bucais, especificamente oriundas da osteoporose senil, no intuito de abordar os problemas odontológicos que podem ser gerados no público idoso por si só, que já são muitos e tendem a ser aumentados quando da presença concomitante da osteoporose senil, podendo entre outras ocorrências afligir a ATM, causando DTM e DOF.

O idoso requer avaliação global, que exige a atenção de diversas especialidades médicas e odontológicas, englobando Geriatria e a Odontogeriatria, entre outras, tanto pelo processo fisiológico de envelhecimento, como por ocorrerem alterações sistêmicas múltiplas (VERAS, R, 2009).

Faz parte de uma anamnese completa a identificação de fatores predisponentes, que aumentam o risco da DTM; fatores iniciadores, que causam a instalação das DTMs e fatores perpetuantes, que interferem no controle da patologia. Dentre esses fatores possuem relevância, os fatores fisiopatológicos, através dos fatores sistêmicos envolvidos, como: doenças degenerativas, endócrinas, infecciosas, metabólicas, neoplásicas, neurológicas, vasculares e reumatológicas (LEEUW, R, 2010).

Condições sistêmicas podem ter forte impacto na função e saúde oral. Entendese por saúde oral, a ausência de doenças dentárias e gengivais, dor orofacial e a presença de função mandibular adequada. Corresponde a um quadro de ausência de algumas anormalidades na cavidade bucal, como: dor crônica facial e na boca, cancro oral e da garganta, feridas orais, defeitos congênitos (lábio e/ou fenda palatina), doença periodontal, perdas dentárias, bem como abrange a inexistência de outras doenças e pertubações orais que possam afetar a cavidade oral e a boca (WHO, 2007).

A osteoporose manifesta-se a princípio sistemicamente, podendo apresentar repercussões na cavidade oral à medida que a doença avança. Quanto antes houver sua descoberta e tratamento, melhor será seu prognóstico, minimizando-se os danos ou sequelas, comumente fraturas, que podem ser causados, mantendo-se a doença sob controle médico e/ou farmacológico. 
Sabe-se que a osteoporose não possui cura e que essa doença permanece apenas sob controle terapêutico. Trata-se de doença que pode se manifestar em qualquer idade, inclusive na infância e na adolescência (SPEZZIA, S, 2017).

A relação da osteoporose sistêmica com a osteoporose oral é um problema complexo, sendo motivo de um grande número de pesquisas experimentais e clínicas (WHITE, SC et al., 2005).

As DTMs são caracterizadas por um conjunto de condições médicas e dentárias que afetam a ATM, músculos mastigatórios e tecidos adjacentes, sendo a condição de dor orofacial crônica, a mais enfrentada por cirurgiões dentistas e outros profissionais da saúde (NÓBREGA, JCM et al., 2007; SILVEIRA, AM et al., 2007).

Em alguns casos, doenças sistêmicas podem ser a causa de DTMs e assim dificultar o verdadeiro diagnóstico de base. O papel do cirurgião dentista na análise semiótica frente as DTMs, deve abranger o conhecimento de seus principais sintomas, daqueles relacionados a doenças sistêmicas e a quadros sintomatológicos de áreas anexas. O profissional deve atentar para as manifestações sistêmicas comuns de dor crônica para que possa formular o diagnóstico diferencial e estabelecer a conduta terapêutica adequada.

Para que o tratamento protético seja realizado com sucesso em idosos é importante verificar se o paciente é portador de alguma doença sistêmica, envolvendo possivelmente osteoporose, diabetes, artrite, problemas cardiovasculares, entre outras, que possam interferir no tratamento. Deve-se ainda avaliar as medicações de que o paciente faz uso para o controle dessas doenças, uma vez que as interações medicamentosas exercem grande influência no fluxo salivar e podem causar repercussões na cavidade bucal com redução na produção da quantidade normal de saliva e ressecamento das mucosas que irão sustentar as bases das próteses, causando traumas. Com o fluxo salivar normal, as mucosas são lubrificadas fisiologicamente pela ação protetora da saliva. Os bisfosfonatos por exemplo, são prescritos comumente no tratamento da osteoporose e devem ser considerados na avaliação (BORAKS, S, 2002).

\section{CONCLUSÃO}


Concluiu-se que a osteoporose pode influir nas manifestações clínicas das DOF, sendo fundamental o conhecimento e a conscientização pelo cirurgião dentista dessa doença sistêmica que pode acometer a saúde bucal dos seus pacientes.

\section{REFERÊNCIAS}

1. The WHO Study Group. Assessment of fracture risk and its application to screening for postmenopausal osteoporosis. Switzerland: World Health Organization, 1994.

2. National Osteoporosis Foundation. Clinician's guide to prevention and treatment of osteoporosis. Washington (DC): NOF, 2010 [cited 2011 Mar 15]. Available from: http://www.nof.org/sites/default/files/pdfs/NOF_ClinicianGuide2009_v7.pdf.

3. Siqueira JTT. Disfunção temporomandibular: classificação e abordagem clínica. In: Siqueira JTT, Teixeira MJ. Dor orofacial: diagnóstico, terapêutica e qualidade de vida. $1^{\mathrm{a}}$. ed. Curitiba: Ed. Maio, 2001.

4. Siqueira JTT. As consequências das dores orofaciais para a saúde. J Dor, 2005; 18:3.

5. Siqueira, JTT, Teixeira MJ. Dor orofacial e disfunção temporomandibular: abordagem clínica atual. JBO, 1998; 17(73):36-50.

6. Mohl ND, Zarb GA, Carlsson GE, Rugh JD. Fundamentos de oclusão. $2^{\mathrm{a}}$ ed. Rio de Janeiro: Quintessence, 1991.

7. Molina OF. Fisiopatologia Craniomandibular (Oclusão e ATM). 2a . Ed. São Paulo: Pancast, 1989.

8. Turp JC, Kowalski CJ, Stohler CS. Temporomandibular disorders - pain outside the head and face is rarely acknowledged in chief complaint. J Prosthet Dent, 1997; 78(6):592-5.

9. Klineberg I, McGregor N, Butt H, Dunstan H, Roberts T, Zerbes M. Chronic orofacial muscle pain: a new approach to diagnosis and management. Alpha Omegan, 1998. 91(2):25-8.

10. Spezzia S. Saúde bucal em idosos com osteoporose: relato de casos. Rev Uningá Review, 2013; 16(1):64-9.

11. Pedroso RA, Celich KLS. Dor: quinto sinal vital, um desafio para o cuidar em enfermagem. Texto Contexto Enferm, 2006; 15(2):270-6. 
12. Cohen LL, Lemanek K, Blount RL, Dahlquist LM, Lim CS, Palermo TM et al. Evidence-based assessment of pediatric pain. J Pediatr Psychol, 2008; 33(9):939-55.

13. Tommasi AF. Diagnóstico em Patologia Bucal. $3^{\mathrm{a}}$ ed. São Paulo: Ed. Pancast Editorial, 2002.

14. Lobo LF, Nunes LJ. Tratamento Cirúrgico. ATM - Diagnóstico e tratamento. São Paulo: Pancast, 2000.

15. Spezzia S. Reparação óssea alveolar, metabolismo do cálcio, fósforo e osteoporose. Rev Uningá Review, 2013; 13(1):136-42.

16. Spezzia S. O papel da osteoporose na odontologia. Rev Assoc Paul Cir Dent, 2014; 68(4):366-7.

17. Spezzia S. Inter-relação entre osteoporose e doenças periodontais. Rev Implant News Perio, 2016; 1(6):1207-13.

18. Spezzia S. Manifestações ósseas bucais da osteoporose. Rev Cienc Med, 2017; 26(2):67-76.

19. Dervis E. Oral implications of osteoporosis. Oral Surg Oral Med Oral Pathol Oral Radiol Endod, 2005; 100(3):349-56.

20. Siqueira JTT, Jacobsen MT. Dor Orofacial, Diagnóstico, Terapêutica e Qualidade de Vida. $1^{\text {a }}$. Ed. Curitiba: Ed. Maio, 2001.

21. Barros LF. ATM: articulação temporomandibular. 2004. Disponível em: http://www.atm.hostmidia.com.br/atm2.html. Acessado em 13 de maio 2018.

22. Veras R. Envelhecimento populacional contemporâneo: demandas, desafios e inovações. Rev Saúde Pública, 2009; 43(3):548-54.

23. Leeuw R. Dor orofacial: guia de avaliação, diagnóstico e tratamento. $4^{\mathrm{a}}$. ed. São Paulo: Quintessence, 2010.

24. World Health Organization. Oral health. WHO, 2007. Acessado em 20 de Julho de 2017 Disponível em: http://www.who.int/mediacentre/factsheets/fs318/en/index.html 25. Spezzia S. A Osteoporose na Infância e na Adolescência. Ensaios Cienc Cienc Biol Agrar Saúde, 2017; 21(3):142-7.

26. White SC, Taguchi A, Kao D, Wu S, Service SK, Yoon D et al. Clinical and panoramic predictors of femur bone mineral density. Osteoporos Int, 2005; 16(3):33946.

REVISTA FLUMINENSE DE ODONTOLOGIA - ANO XXVI - No 53 - Janeiro / Julho 2020 
27. Nóbrega JCM, Siqueira SRDT, Siqueira JTT, Teixeira MJ. Differential Diagnosis in Atypical Facial Pain. Arq Neuropsiquiatr, 2007; 65(2-A):256-61.

28. Silveira AM, Feltrin PP, Zanetti RV, Mantoni MC. Prevalência de portadores de DTM em pacientes avaliados - setor de otorrinolaringologia. Rev Bras Otorrinolaringol, 2007; 7(3):528-32.

29. Boraks S. Distúrbios bucais na terceira idade. In: Brunetti RF, Montenegro FLB. Odontogeriatria: noções de interesse clínico. 1ª Ed. São Paulo: Artes Médicas, 2002. 